\title{
An Anatomical Equivalence Class Based Joint Transformation-Residual Descriptor for Morphological Analysis
}

\author{
Sajjad Baloch, Ragini Verma, and Christos Davatzikos \\ University of Pennsylvania, Philadelphia, PA
}

\begin{abstract}
Existing approaches to computational anatomy assume that a perfectly conforming diffeomorphism applied to an anatomy of interest captures its morphological characteristics relative to a template. However, biological variability renders this task extremely difficult, if possible at all in many cases. Consequently, the information not reflected by the transformation, is lost permanently from subsequent analysis. We establish that this residual information is highly significant for characterizing subtle morphological variations and is complementary to the transformation. The amount of residual, in turn, depends on transformation parameters, such as its degree of regularization as well as on the template. We, therefore, present a methodology that measures morphological characteristics via a lossless morphological descriptor, based on both the residual and the transformation. Since there are infinitely many [transformation, residual] pairs that reconstruct a given anatomy, which collectively form a nonlinear manifold embedded in a high-dimensional space, we treat them as members of an Anatomical Equivalence Class (AEC). A unique and optimal representation, according to a certain criterion, of each individual anatomy is then selected from the corresponding AEC, by solving an optimization problem. This process effectively determines the optimal template and transformation parameters for each individual anatomy, and removes respective confounding variation in the data. Based on statistical tests on synthetic 2D images and real 3D brain scans with simulated atrophy, we show that this approach provides significant improvement over descriptors based solely on a transformation, in addition to being nearly independent of the choice of the template.
\end{abstract}

\section{Introduction}

The problem of quantitatively characterizing normal and pathologic anatomy is usually addressed in computational anatomy through high dimensional diffeo-

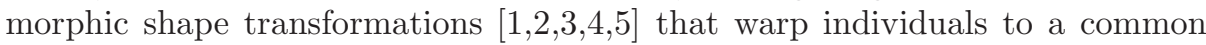
template and vice-versa. Various descriptors are then derived from these transformations for quantifying morphological characteristics, leading to a wide range of approaches such as deformation based morphometry (DBM) 678, voxel based morphometry (VBM) 91011, and tensor based morphometry (TBM) [1213. Accuracy of these methods, however, depends on the ability to establish perfect correspondences between subjects and a template, which may, in general, not be 
possible due to anatomical variability across individuals. For instance, a biologically meaningful diffeomorphism that warps a single-folded sulcus to a bifolded sulcus may not exist. As a result, a shape transformation would likely fail to capture the shape of the sulcus, resulting in the loss of residual information. In this paper, we establish that the residual may carry more important information than the transformation itself, and, therefore, consider a complete morphological descripton (CMD) of the form [Transformation, Residual].

CMD requires additional consideration due to its dependence on transformation parameters such as the degree of conformity achieved by the transformation, which is determined by some regularization parameter(s), as well as on the selection of a template. Since the selection of these parameters is an open question in computational anatomy, a complete representation does not lead to a unique descriptor. By varying these parameters, one may, in fact, generate an entire class of infinitely many anatomically equivalent descriptors, all representing the same anatomy via different transformations and respective residuals. This class, the Anatomical Equivalence Class (AEC), is generally a nonlinear manifold embedded in a high dimensional space. In this paper, we address the problem of non-uniqueness of this anatomical descriptor by representing each anatomy with an "optimal" descriptor, referred to as Optimal Morphological Signature (OMS), which is estimated by minimizing the variance of an ensemble, while allowing each CMD to slide along its respective AEC. The hypothesis is that by minimizing the variance due to confounding factors, one may overcome arbitrariness in selecting these parameters, thereby enhancing the ability to detect morphological abnormalities. Our results indicate that this morphological representation provides generally better and more robust detection of group morphological differences than the transformation alone. Moreover, it is nearly invariant to template selection, and the amount of flexibility in the transformation, thus avoiding their arbitrary selection.

\section{Morphological Descriptor Framework}

\subsection{Motivation}

Computational anatomy typically involves characterizing anatomical differences between a subject $S$ and a template $T$ by mapping the template space $\Omega_{T}$ to the subject space $\Omega_{S}$ through a diffeomorphism $h \in \mathcal{H}_{S}: \Omega_{T} \rightarrow \Omega_{S}, \mathbf{x} \mapsto h(\mathbf{x})$, where $\mathcal{H}_{S}$ is the set of all diffeomorphic transformations that maximize some similarity criterion between $T$ and normalized subject $S_{T}$. Resulting transformation $h$ then carries information about morphological differences between a subject and a template 6 2 7/14/912/0|8/113]. This, however, requires a diffeomorphism that perfectly warps a subject to the template, i.e., $T(\mathbf{x})-S(h(\mathbf{x}))=0, \forall \mathbf{x} \in \Omega_{T}$. Moreover, $h$ must define anatomical correspondences, instead of simply matching

${ }^{1}$ The term "complete" is used, since this morphological descriptor does not discard any image information. 


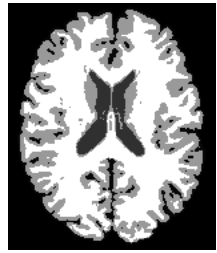

(a)

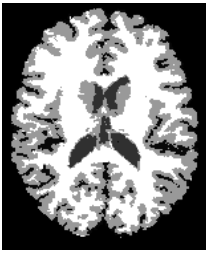

(b)

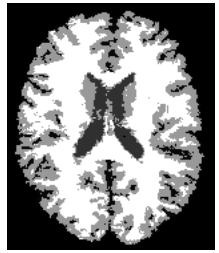

(c)

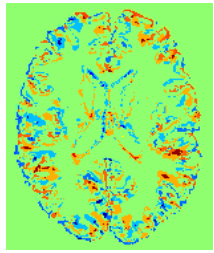

(d)

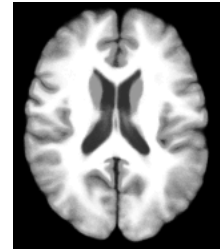

(e)

Fig. 1. (a) Template; (b) A representative subject; (c) Spatial normalization of (b); (d) Corresponding residual; (e) Crispness of the mean of 31 spatially normalized subjects indicates relatively good anatomical correspondence

images. Achieving both of these goals is usually not possible, thereby resulting in a residual error:

$$
R_{h}(\mathbf{x}):=T(\mathbf{x})-S(h(\mathbf{x})), \mathbf{x} \in \Omega_{T}
$$

This is shown with an illustrative example in Fig. 1] based on the spatial normalization of 31 subjects using a deformable registration method 5 . While the accuracy of the registration is demonstrated by the clarity of the average brain given in Fig. 1(e), spatial normalization of a typical subject (Fig. 1(b)) to the template (Fig. 1(a)) still produces significant residual shown in Fig. 1(d). A study based solely on a warping transformation irreversibly loses this residual information. Although one may argue that a more flexible and aggressive registration may further minimize the residual, this is often impossible to achieve using biologically consistent transformations. Our approach herein is to use a template warping algorithm that is flexible enough, yet respecting anatomical correspondences and then capture the resulting residual in an $\mathcal{M}_{h}:=\left(h, R_{h}\right)$ descriptor, referred to as CMD.

\subsection{Anatomical Equivalence Class Framework}

$\mathrm{CMD}, \mathcal{M}_{h}$, defined in the previous section not only depends on the underlying anatomy but also on transformation parameters, resulting in a non-unique representation of the anatomy. An entire family of anatomically equivalent CMDs may be generated by varying $h \in \mathcal{H}_{S}$. This unwanted variation usually confounds subsequent analyses, and, therefore, must be eliminated. We approach this problem by first approximating the range of variation in $\mathcal{M}_{h}$. Since it is not possible to sample all possible transformations and to evaluate their respective residuals for all possible templates, we examine a restricted family of transformations $h_{\lambda, \tau} \in \mathcal{I}_{S} \subset \mathcal{H}_{S}$, by perturbing $h_{\lambda, \tau}$ through two parameters, namely $\lambda \in \mathbb{R}_{+}$ and $\tau \in \mathcal{T}^{m}$, where $\mathcal{T}$ represents the set of all possible templates. The first parameter $\lambda$ represents the level of regularity (smoothness) in $h_{\lambda, \tau}$, as determined by viscosity in viscous fluid registration, for instance, or Young's modulus in elastic registration. For notational consistency, we reparameterize the regularization parameter such that small $\lambda_{\mathrm{s}}$ correspond to aggressive transformations, and, hence, 


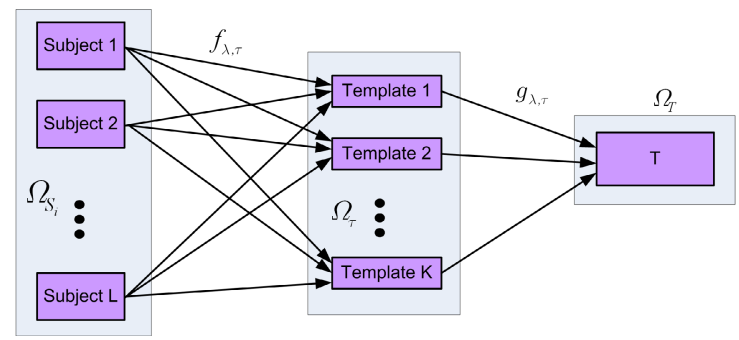

Fig. 2. Constructing AECs: Each subjects is normalized to $\Omega_{T}$ via intermediate templates at different smoothness levels of the warping transformations

small residuals. The second parameter, $\tau$, denotes a sequence of $m$ intermediate templates, $\tau:=\tau_{1} \rightarrow \ldots \rightarrow \tau_{m}$, between $S$ and $T$. For a given smoothness, $\lambda$, individual anatomies are first normalized to intermediate templates $\tau$ through $f_{\lambda, \tau} \in \mathcal{F}_{S}$ as shown in Fig. 2. Intermediate results are then warped to $T$ through $g_{\lambda, \tau} \in \mathcal{G}_{S}$, since we ultimately need to go to a single reference space, $\Omega_{T} . \mathcal{F}_{S}$ and $\mathcal{G}_{S}$ respectively denote sets of all $\tau-S \circ f_{\lambda, \tau}$ and $T-\left(S \circ f_{\lambda, \tau}\right) \circ g_{\lambda, \tau}$ minimizing diffeomorphisms. As a result $h_{\lambda, \tau}:=f_{\lambda, \tau} \circ g_{\lambda, \tau}$ takes $S$ to the template $T$, with $\tau$ perturbing the path taken by $h_{\lambda, \tau}$ from an individual to the template by providing a sequence of milestones that may facilitate registration. A poor choice of intermediate templates increases the amount of the residual, whereas appropriately selected intermediate templates may find paths for the transformation $h_{\lambda, \tau}$ that result in minimal residuals. In many instances, a different choice of $T$ may result in different registration results, as shown in Fig. 3(d) and (e). This inconsistency will be eliminated in subsequent formulation by viewing how registration responds to different intermediate templates. In short, introduction of $\tau$ and $\lambda$ provides variability in $\left(h_{\lambda, \tau}, R_{h_{\lambda, \tau}}\right)$ necessary to account for template and parameter bias, which allows us to consider the restriction of $\mathcal{H}_{S}$ :

$$
\mathcal{I}_{S}:=\left\{h_{\lambda, \tau}=f_{\lambda, \tau} \circ g_{\lambda, \tau} \in \mathcal{H}_{S}: g_{\lambda, \tau} \in \mathcal{G}_{S}, f_{\lambda, \tau} \in \mathcal{F}_{S}, \lambda \in \mathbb{R}_{+}, \tau \in \mathcal{T}^{m}\right\},
$$

for subsequent analysis. The anatomies, which are otherwise not well represented by the template $T$, may be captured effectively through $\mathcal{I}_{S}$ (Fig. 4). By introducing this variability in $\left(h_{\lambda, \tau}, R_{h_{\lambda, \tau}}\right)$ carefully restricted to $\lambda$ and $\tau$, we generate a class of equivalent anatomical representations, $\mathcal{A}(S)=\left\{\left\{\mathcal{M}_{h}(\mathbf{x}): S(h(\mathbf{x}))=\right.\right.$ $\left.\left.T(\mathbf{x})-R_{h}(\mathbf{x}), \forall \mathbf{x} \in \Omega_{T}\right\}, \forall h \in \mathcal{I}_{S}\right\}$, referred to as AEC. For notational simplicity, we combine the two parameters, $\tau$ and $\lambda$, in $\boldsymbol{\theta}$ to represent AEC as:

$$
\mathcal{A}(S)=\left\{\left\{\left(\mathcal{M}_{h_{\boldsymbol{\theta}}}(\mathbf{x})\right): S\left(h_{\boldsymbol{\theta}}(\mathbf{x})\right)=T(\mathbf{x})-R_{h_{\boldsymbol{\theta}}}(\mathbf{x}), \forall \mathbf{x} \in \Omega_{T}\right\}, \forall \boldsymbol{\theta} \in \boldsymbol{\Theta}\right\},
$$

where $\mathcal{M}_{h_{\boldsymbol{\theta}}}(\mathbf{x})$ denotes CMD $\left(h_{\boldsymbol{\theta}}(\mathbf{x}), R_{h_{\boldsymbol{\theta}}}(\mathbf{x})\right)$. An AEC maps each anatomy to a (generally nonlinear) manifold in an $n$-dimensional space as shown schematically in Fig. 5(a), where $n$ is the dimensionality of CMD. Although the resulting AEC is rich with an entire range of variability in $R_{h_{\theta}}$, one may not arbitrarily select $\boldsymbol{\theta}$ 


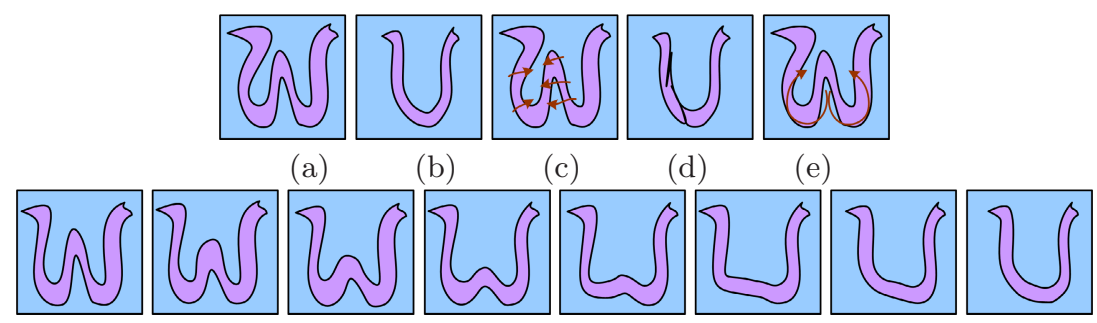

(f)

Fig. 3. (a) Template; (b) Subject; (c) Deformation field for direct warping - middle of $\mathrm{W}$ is mapped to a limb of V; (d) Warped subject; (e) Sequence of transformations obtained through intermediate templates of (f) may open up a W-fold to a V-fold by guiding registration; (f) Intermediate templates for (e)

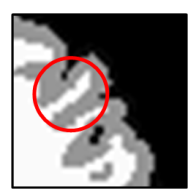

(a)

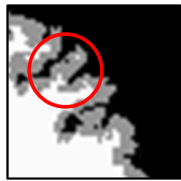

(b)

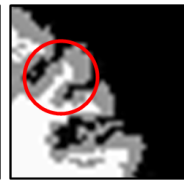

(c)

Fig. 4. Intermediate templates aid registration: (a) Template; (b) Direct warping of Fig. 1(c); (c) Warping via an intermediate template

for analysis. It should be noted that a small residual does not necessarily correspond to the best CMD. For instance, the residual may be reduced by selecting small $\lambda \mathrm{s}$, but it may lead to an overly aggressive registration, which introduces noise and erroneous correspondence as shown in Fig. 3(d), where viscous fluid based registration was employed [1]. It is, therefore, important to find the optimal parameter selection, which requires a metric for carrying out intersubject comparisons based on AEC manifolds. One may define distance between two anatomies as infimum separation between their anatomical manifolds as shown in Fig. 5(b), i.e., $\operatorname{Dist}\left(S_{A}, S_{B}\right):=\inf \left(\left\{d\left(\mathcal{M}_{h_{A}}^{A}, \mathcal{M}_{h_{B}}^{B}\right): \forall h_{A} \in \mathcal{F}_{S_{A}}, \forall h_{B} \in \mathcal{F}_{S_{B}}\right\}\right)$, where $d$ represents Euclidean distance defined on the space of CMDs. While this works for two subjects, comparing three or more anatomies becomes problematic as illustrated in the figure, where $\operatorname{Dist}\left(S_{A}, S_{C}\right) \not \leq \operatorname{Dist}\left(S_{A}, S_{B}\right)+\operatorname{Dist}\left(S_{B}, S_{C}\right)$, which is no longer a metric. We circumvent this problem by representing each AEC through OMS, which allows directly employing a Euclidean metric for groupwise comparisons.

Since we are mainly interested in volumetric variations among subjects representing tissue growth or death, in subsequent discussion, we utilize Jacobian determinant (JD), $J_{h_{\boldsymbol{\theta}}}$, of $h_{\boldsymbol{\theta}}$ to construct the proposed AEC through $\mathcal{M}_{h_{\boldsymbol{\theta}}}=\left(J_{h_{\boldsymbol{\theta}}}, R_{h_{\boldsymbol{\theta}}}\right)$ descriptors. Moreover, we consider a single milestone $(m=1)$ between $S$ and $T$, with $\tau$ representing a single intermediate template in $\mathcal{T}$. 


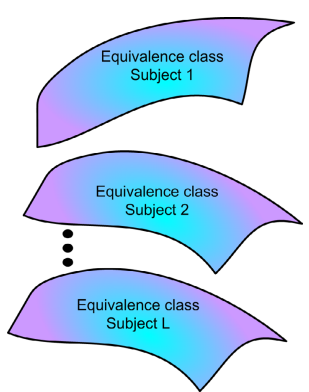

(a)

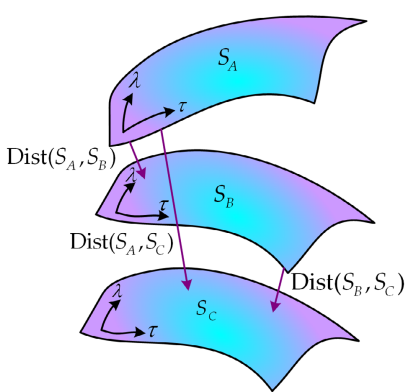

(b)

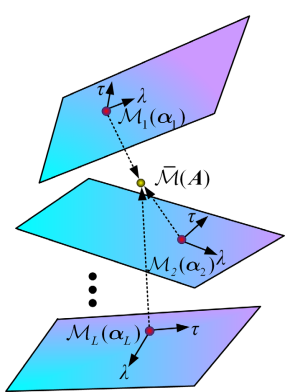

(c)

Fig. 5. (a) Nonlinear manifold structure of AEC; (b) Problem with Euclidean distance based comparisons; (c) Approximation with hyperplanes

The idea of AEC was first proposed in [15], where tissue density maps [10] were employed with $\lambda$ as the sole confounding factor. Its major limitation was the assumption that all AECs were collinear and could be approximated by a single hyperplane. We extend this idea by providing a robust way to eliminate non-uniqueness of CMDs and present a general framework that incorporates all confounding factors in $\boldsymbol{\theta}$.

\subsection{Optimal Morphological Signature}

Optimal choice of $\boldsymbol{\theta}$ for one subject may be different from that for the others, which intuitively follows from the fact that different anatomies are affected differently by it. For instance, a subject with bifolded sulci will prefer similar structures in the template. It is, therefore, imperative to find an appropriate $\tau$ and $\lambda$ for each subject that is optimized for the underlying anatomy, leading to optimal parameters $\boldsymbol{\Theta}=\left(\boldsymbol{\theta}_{1}, \ldots, \boldsymbol{\theta}_{L}\right)$ for $L$ subjects.

In order to define our criterion for optimality of $\Theta$, we first consider two anatomies. If we were to slide along the respective AECs, we would find two representations that have minimum distance. We consider these two representations as the ones that best highlight differences between the two anatomies, since together they eliminate confounding effects of $\lambda$ and $\tau$. More generally, for $L$ anatomies, we allow their representations to slide along respective AECs in order to minimize the cumulative of pairwise distances of all individuals:

$$
\boldsymbol{\Theta}^{*}=\arg \min _{\substack{\boldsymbol{\Theta}=\left(\boldsymbol{\theta}_{1}, \ldots, \boldsymbol{\theta}_{L}\right) \\ \mathcal{M}_{k}\left(\boldsymbol{\theta}_{k}\right) \in \mathcal{A}\left(S_{k}\right), k=1, \ldots, L}} \sum_{i=1}^{L} \sum_{\substack{j=1 \\ j \neq i}}^{L} d^{2}\left(\mathcal{M}_{i}\left(\boldsymbol{\theta}_{i}\right), \mathcal{M}_{j}\left(\boldsymbol{\theta}_{j}\right)\right),
$$

where $d$ denotes the Euclidean distance, and $\mathcal{M}_{i}\left(\boldsymbol{\theta}_{i}\right)=\left(J_{h_{\boldsymbol{\theta}_{i}}}, R_{h_{\boldsymbol{\theta}_{i}}}\right)$ is the CMD of subject $i$ at the correspondence $\boldsymbol{\Theta}=\left(\boldsymbol{\theta}_{1}, \ldots, \boldsymbol{\theta}_{L}\right)$. It can be shown that the criterion of Eq. (3) minimizes the variance of morphological descriptors over entire ensemble w.r.t. confounding factors, leading to: 


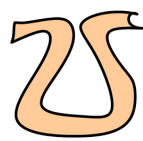

(a)

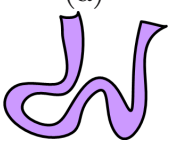

(d)

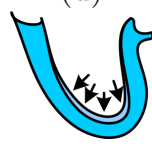

(g)

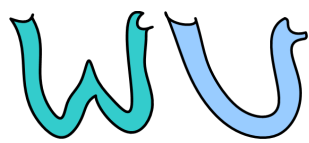

(b)

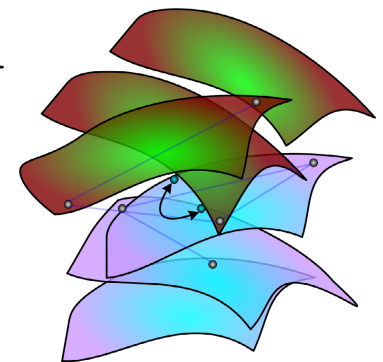

(a)

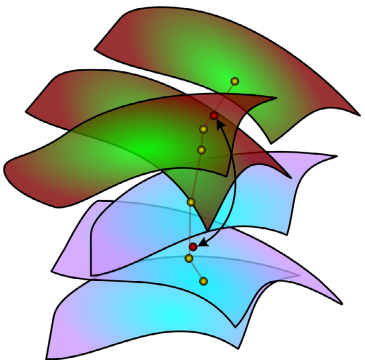

(b)
Fig. 6. (a)-(d) Normalizing templates $\quad T_{1}, \ldots, T_{4} ; \quad$ (e)-(f) Subjects $S_{1}$ and $S_{2} ;(\mathrm{g})-(\mathrm{h})$ Deformation fields for $S_{1}$ and $S_{2}$.
Fig. 7. OMS versus CMD: (a) Randomly selecting CMDs (random parameter selection) from AECs reduces the inter-group separation. Dots marked with arrows represent group means; (b) OMS results in optimal separation between the two groups.

$$
\boldsymbol{\Theta}^{*}=\arg \min _{\substack{\boldsymbol{\Theta}=\left(\boldsymbol{\theta}_{1}, \ldots, \boldsymbol{\theta}_{L}\right) \\ \mathcal{M}_{k}\left(\boldsymbol{\theta}_{k}\right) \in \mathcal{A}\left(S_{k}\right), k=1, \ldots, L}} \sum_{i=1}^{L} d^{2}\left(\mathcal{M}_{i}\left(\boldsymbol{\theta}_{i}\right), \overline{\mathcal{M}}(\boldsymbol{\Theta})\right),
$$

where $\overline{\mathcal{M}}(\boldsymbol{\Theta})=\frac{1}{L} \sum_{i=1}^{L} \mathcal{M}_{i}\left(\boldsymbol{\theta}_{i}\right)$ represents the mean descriptor. The resulting OMS, $\mathcal{M}_{i}\left(\boldsymbol{\theta}_{i}^{*}\right)$, corresponding to optimal parameters, $\boldsymbol{\theta}_{i}^{*}$, for each individual, effectively allows us to select optimal intermediate template and transformation individually for each anatomy. This is a very important aspect of our approach, which is better explained with a schematic example given in Fig. 6. Suppose $S_{1}$ and $S_{2}$ are two anatomies under study, and $T_{1}, \ldots, T_{4}$ are the normalizing templates. Eq. (44) finds for each individual corresponding templates such that the resulting (JD, residual) are as similar as possible. Resultantly, $T_{3}$ and $T_{1}$ will be selected for $S_{1}$ and $S_{2}$ respectively, since they yield very similar transformations and residuals. Selecting a different template, on the other hand, results in dissimilar transformations, and possibly dissimilar residuals (depending on the smoothness level). When generalized to $L$ subjects and $\mathcal{T}$, Eq. (4) yields optimal transformation parameters for each subject as shown Fig. 7 . An analysis based on random CMD selection results in poor inter-group separation (Fig. 7(a)). Eq. (4), on the other hand, helps in removing this randomness of CMDs to provide an optimal separation between the two groups (Fig. 7(b)). Additionally, by constraining each OMS to its respective AEC, it preserves inter-subject and inter-group anatomical differences.

In order to solve the optimization problem of Eq. (4), we need to define the structure of AEC manifolds shown in Fig. 5(a). For simplicity we approximate the AECs with hyperplanes as shown in Fig. 5(c). If $\left\{\mathbf{V}_{j}^{(i)}, j=1, \ldots, n\right\}$ are 
the principal directions of the AEC of $i$ th subject, and $\hat{\mathcal{M}}_{i}$ is the corresponding mean, then the linear hyperplane approximating the corresponding AEC manifold is given by:

$$
\mathcal{M}_{i}(\boldsymbol{\theta})=\hat{\mathcal{M}}_{i}+\sum_{j=1}^{n} \alpha_{i j} \mathbf{V}_{j}^{(i)},
$$

where $\alpha_{i j}, j=1, \ldots, n$ capture transformation dependent parameters, $\boldsymbol{\theta}_{i}$. The objective function of Eq. (4), therefore, becomes:

$$
\boldsymbol{A}^{*}=\arg \min _{\boldsymbol{A}:=\left(\boldsymbol{\alpha}^{1}, \ldots, \boldsymbol{\alpha}^{L}\right)} \sum_{i=1}^{L} d^{2}\left(\hat{\mathcal{M}}_{i}+\sum_{j=1}^{n} \alpha_{i j} \mathbf{V}_{j}^{(i)}, \overline{\mathcal{M}}(\boldsymbol{A})\right),
$$

where:

$$
\overline{\mathcal{M}}(\boldsymbol{A})=\frac{1}{L} \sum_{i=1}^{L}\left(\hat{\mathcal{M}}_{i}+\sum_{j=1}^{n} \alpha_{i j} \mathbf{V}_{j}^{(i)}\right),
$$

is the mean descriptor of all subjects, and $\boldsymbol{\alpha}^{i}=\left(\alpha_{i 1}, \ldots, \alpha_{i n}\right)$. In order to stay on individual manifolds, the optimization is constrained to $\boldsymbol{\alpha}_{i}^{\min } \leq \boldsymbol{\alpha}_{i} \leq \boldsymbol{\alpha}_{i}^{\max }$, where $\boldsymbol{\alpha}_{i}^{\min }$ and $\boldsymbol{\alpha}_{i}^{\max }$ depend on the corresponding principal modes.

Solution to this constrained problem is an algorithm that allows moving along individual hyperplanes, in order to minimize the objective function. At each optimization iteration, an update of $\mathcal{M}_{i}\left(\boldsymbol{\alpha}_{i}\right)$ (Fig. [5.5(c)) is computed, which yields the current floating mean $\overline{\mathcal{M}}(\boldsymbol{A})$ given by Eq. (7). The procedure is repeated until the minimum of Eq. (6) is attained. Analytically it leads to the following solution subject to constraints given above:

$$
\boldsymbol{\alpha}_{i}^{*}=\mathbf{V}^{(i)^{T}}\left[\frac{1}{L-1} \sum_{k \neq i}\left(\hat{\mathcal{M}}_{k}+\mathbf{V}^{(k)} \boldsymbol{\alpha}_{k}\right)-\hat{\mathcal{M}}_{i}\right] \quad i=1, \ldots, L .
$$

When combined with Eq. (5), optimal correspondence of Eq. (8) yields OMS, $\mathcal{M}_{i}^{*}$, which is then used for subsequent analysis. The first term within braces represents the mean across a smaller subset of subjects excluding $i$ th subject. $\boldsymbol{\alpha}_{i}^{*}$ is, therefore, the projection to the principal axes of the difference between the mean across other users and the subject mean. Eq. (8), hence, presents a direct way of estimating the optimal signatures.

OMS, $\mathcal{M}_{i}^{*}$, provides the optimal combination of JD and the corresponding residual in addition to optimal selection of transformation parameters, $\lambda$ and $\tau$, which, herein, makes subsequent analysis invariant to transformation parameters.

\section{Experiments}

In this section, we provide experimental results to support our hypothesis that residual carries significant amount of information for identifying group differences and that OMS yields superior performance by maintaining group separation between normal and pathologic anatomies. We considered two datasets: 


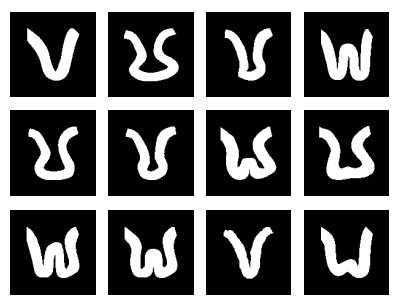

Fig. 8. Templates $T_{1}, \ldots, T_{12}$ simulating gray matter folds

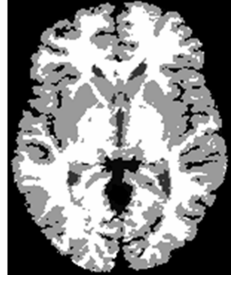

(a)

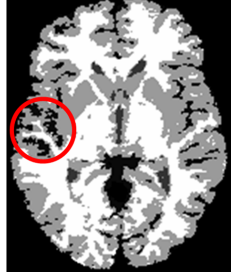

(b)
Fig. 9. (a) A subject without atrophy; (b) With $10 \%$ simulated atrophy

1. A $2 \mathrm{D}$ dataset of 60 subjects was generated by introducing random variability in 12 manually created templates given in Fig. 8. This variability across subjects is close to anatomical differences encountered in real gray matter folds. $5 \%$ atrophy was introduced in center one-third of the fold of 30 subjects to simulate patient data. All subjects were spatially normalized to $T_{12}$ via $T_{1}, \ldots, T_{11}$, for smoothness levels of $\lambda=0, \ldots, 42$ to construct individual AECs.

2. The second dataset consisted of real MRI scans of 31 subjects. To simulate patient data, $10 \%$ atrophy was introduced in 15 randomly selected subjects in a spherical region as shown in Fig. 9] using the simulator of [16. Five intermediate templates were selected for spatial normalization to generate AECs for all subjects with smoothing levels of $\lambda=0, \ldots, 7$.

\subsection{Results and Discussion}

For both datasets, we performed two types of tests on CMDs and OMSs: (1) $t$ tests on individual $\log J_{h_{\lambda, \tau}}$ and $R_{h_{\lambda, \tau}}$ components, and (2) $T^{2}$ test on $\left(\log J_{h_{\lambda, \tau}}\right.$, $R_{h_{\lambda, \tau}}$ ) descriptor, for finding significance of differences between healthy and pathologic anatomies. For CMDs, we randomly selected intermediate templates for each subject before conducting tests for all smoothness levels $\lambda$. Note that residual was smoothed with a Gaussian filter with various selections of smoothness parameter $\sigma$ prior to statistical tests mainly due to two reasons. First, it ensures the Gaussianity of the smoothed residual. Second, since the residuals appear only on tissue boundaries, even if tissue atrophy is in the interior of the structure, smoothing produces a more spatially uniform residual. JD, on the other hand, was not smoothed for the $T^{2}$ test due to its inherent smoothness.

Based on statistical tests, $p$-value maps were computed for all values of $\sigma$ (and $\lambda$ for tests on CMDs). Since the minimum of a $p$-value map provides the best performance for group differences, we computed minimum of each $p$-value map for both datasets. Minimum $\log _{10} p$ plots as a function of $\sigma$ are given in Figs. 10 and 11 for 2D and 3D datasets respectively. It may be observed from results based on CMDs for both datasets that residual achieves considerably lower $p$ values as compared with JD, which indicates the significance of residual for capturing group differences. The significance of both log JD and the residual increases with 


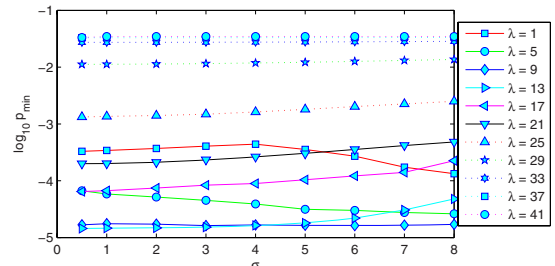

(a)

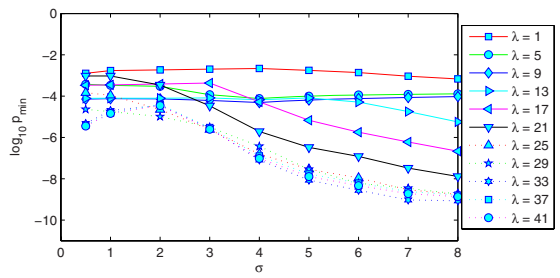

(c)

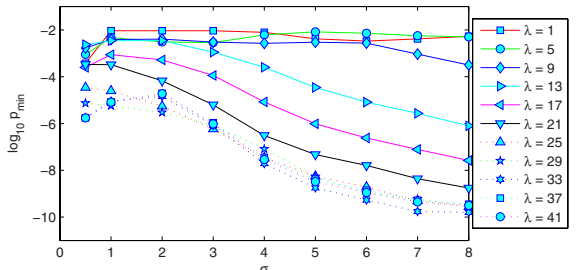

(b)

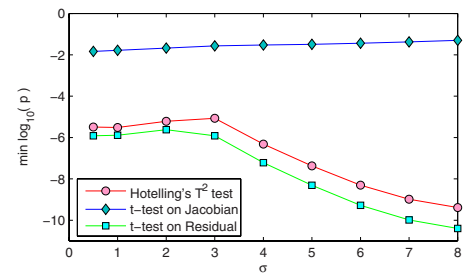

(d)

Fig. 10. Minimum $p$-value plots for synthetic dataset: (a) $t$-test on AEC based log JD; (b) $t$-test on AEC based Residual; (c) $T^{2}$-test on AEC based ( $\left.\log J_{h_{\lambda, \tau}}, R_{h_{\lambda, \tau}}\right)$; (d) Statistical tests on OMS

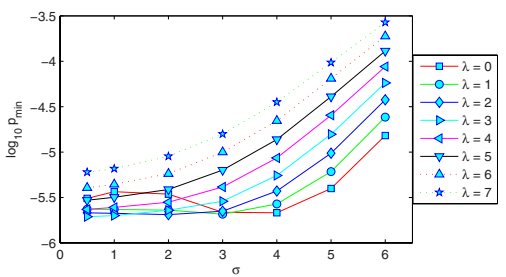

(a)

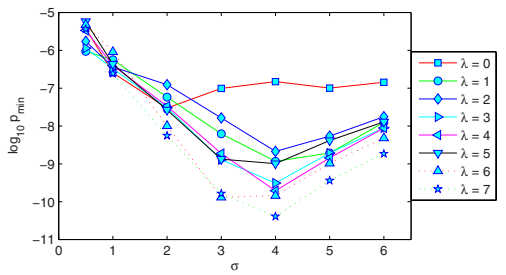

(c)

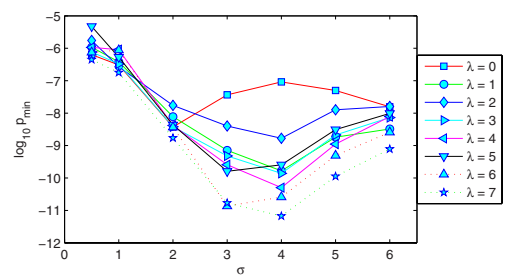

(b)

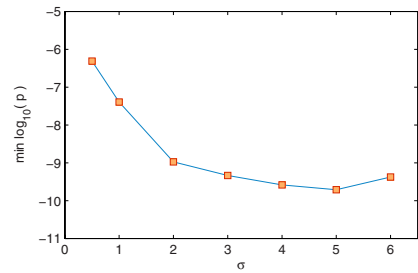

(d)

Fig. 11. Minimum $p$-value plots for real 3D dataset: (a) $t$-test on AEC based $\log J_{h_{\lambda, \tau}}$; (b) $t$-test on AEC based $R_{h_{\lambda, \tau}}$; (c) $T^{2}$-test on AEC based (log $J_{h_{\lambda, \tau}}, R_{h_{\lambda, \tau}}$ ); (d) $T^{2}$-test on OMS

$\sigma$ and $\lambda$ up to a point after which it starts degrading. Similarly, $T^{2}$ test also shows best performance for intermediate values of $\lambda(\lambda=33$ for $2 \mathrm{D}$ and $\lambda=7$ for $3 \mathrm{D})$, which means that an overly aggressive transformation is not required 


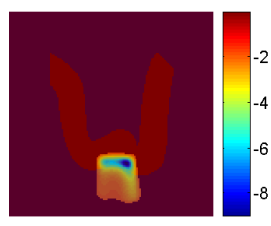

(a)

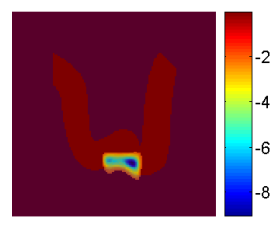

(b)

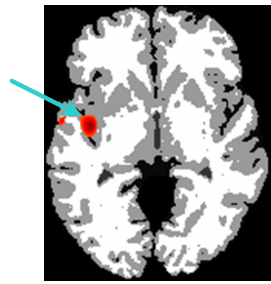

(a)

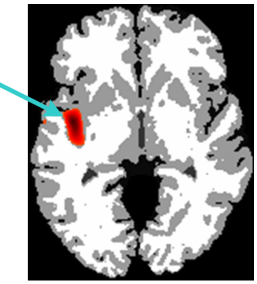

(b)

Fig. 12. $T^{2}$-test based $\log _{10} p$-value maps for 2D simulated data at $\sigma=8$ thresholded to $p \leq 10^{-2}$ : (a) CMD $\lambda=33$; (b) OMS

Fig. 13. $T^{2}$ test based $p$-value maps for $3 \mathrm{D}$ dataset corresponding to best results for each descriptor thresholded to $p \leq 10^{-5}$ : (a) $\mathrm{CMD} \lambda=7, \sigma=4$; (b) OMS with $\sigma=5$; (c) OMS with $\sigma=5$ thresholded to $p \leq 10^{-3}$

for capturing anatomical differences. These observations are in accordance with our hypothesis that residual carries important anatomical information that is complementary to the transformation. An analysis based solely on JD loses this information, thereby degrading the performance of subsequent diagnostic tests. The dependence of $\left(J_{h_{\lambda, \tau}}, R_{h_{\lambda, \tau}}\right)$ on $\lambda$ is eliminated through optimization as indicated by OMSs for $2 \mathrm{D}$, which shows an improved performance versus CMDs ( $p=10^{-9.5}$ at $\sigma=8$ for OMS vs $p=10^{-8.5}$ at $\sigma=8, \lambda=33$ for CMDs). For $3 \mathrm{D}$ dataset, the optimal signatures provide a performance similar to the CMDs, with the latter performing slightly better $\left(p=10^{-9.75}\right.$ against $\left.p=10^{-10.5}\right)$. On the other hand, OMS appears to be relatively insensitive to $\sigma$ with a better dynamic range. For instance, the variation in $p$-values for OMS in the range $\sigma=2-6$ is $10^{-0.75}$, whereas that for CMDs is $10^{3.5}$, which makes CMDs much more sensitive to $\sigma$. Small variations in $\sigma$, therefore, may considerably degrade CMD-based analysis. OMS is, hence, not only more robust but also maintains the separation between the two groups as indicated by very low $p$-values.

Another advantage of OMS may be observed from appropriately thresholded $p$-value maps for 2D and 3D datasets given in Figs. 12 and 13 respectively. Clearly, OMS helps in precisely localizing atrophy, which is in accordance with the objective function of Eq. (6). On the other hand, CMDs fail to localize atrophy, and occasionally result in false positives.

\section{Conclusions}

In this paper, we have presented a morphological descriptor for computational anatomy with two major contributions. First, the transformation was combined with the residual for a complete (lossless) description of the anatomy. Second, each anatomy was represented with a rich class of descriptors, that incorporates 
smoothness of the transformation and the choice of intermediate templates. Independence to these parameters was established through OMS, which was estimated using a manifold sliding algorithm. Since each OMS was forced to stay on the corresponding AEC manifold, it maintains inter-group differences, which was validated through statistical tests on synthetic $2 \mathrm{D}$ as well as real $3 \mathrm{D}$ data.

Tests confirmed our hypothesis that residual provides remarkably better group separation than the JD. Results clearly indicated that residual contains not only important, but critical morphological information, and should, therefore, be an integral part of any morphological descriptor, if not the most important part. Marginal gain was achieved by using the OMS, when compared to the absolutely best result that can be obtained from individual pairs of (transformation, residual). Although this may indicate that the optimization along AEC manifolds could well be omitted, it is argued that the robustness and independence of the results from template and transformation parameters are important reasons to perform this optimization, even if it does not significantly improve detection power at the absolutely optimal set of parameter values. Moreover, future work on estimating the nonlinear structure of these manifolds, instead of approximating them linearly, may further increase the significance of the optimization procedure.

Acknowledgements. This work was supported by the NIH-funded Grant R01AG14971. The authors extend special thanks to Professor Dinggang Shen for interesting and stimulating discussions.

\section{References}

1. Christensen, G., Rabbit, R., Miller, M.: A deformable neuroanatomy textbook based on viscous fluid mechanics. In: Proc. CISS'93 1993, pp. 211-216 (1993)

2. Miller, M., Banerjee, A., et al.: Statistical methods in computational anatomy. Statistical Methods in Medical Research 6, 267-299 1, 2 (1997)

3. Miller, M., Younes, L.: Group actions, homeomorphisms, and matching: a general framework. International Journal of Computer Vision 41(1), 61-84, 1 (2001)

4. Ashburner, J., Friston, K.: Nonlinear spatial normalization using basis functions. Human Brain Mapping 7(4), 254-266, 1 (1999)

5. Shen, D., Davatzikos, C.: HAMMER: Hierarchical attribute matching mechanism for elastic registration. IEEE TMI 21(11), 1421-1439 1,3 (2002)

6. Davatzikos, C., et al.: A computerized approach for morphological analysis of the corpus callosum. Journal of Comp. Assisted Tomography 20(1), 88-97 1, 2 (1996)

7. Ashburner, J., et al.: Identifying global anatomical differences: deformation-based morphometry. Human Brain Mapping 6(6), 348-357 1, 2 (1998)

8. Chung, M., Worsley, K., et al.: A unified statistical approach to deformation-based morphometry. NeuroImage 14(3), 595-600 1, 2 (2001)

9. Ashburner, J., Friston, K.J.: Voxel-based morphometry - the methods. NeuroImage 11(6), 805-821 1, 2 (2000)

10. Davatzikos, C., Genc, A., Xu, D., Resnick, S.: Voxel-based morphometry using RAVENS maps: methods and validation using simulated longitudinal atrophy. Neuroimage 14, 1361-1369 1,2,5 (2001) 
11. Chetelat, G., et al.: Mapping gray matter loss with voxel-based morphometry in mild cognitive impairment. Neuroreport 13(15), 1939-1943 1, 2 (2002)

12. Thompson, P., et al.: Growth patterns in the developing human brain detected using continuum-mechanical tensor mapping. Nature 404(6774), 190-193 1,2 (2000)

13. Leow, A., Klunder, A., et al.: Longitudinal stability of mri for mapping brain change using tensor-based morphometry. Neuroimage 31(2), 627-640 1,2 (2006)

14. Joshi, S.: Large deformation diffeomorphisms and Gaussian random fields for statistical characterization of brain sub-manifolds. PhD thesis, Washington University, St. Louis 2 (1998)

15. Makrogiannis, S., et al.: Anatomical equivalence class: A computational anatomy framework using a lossless shape descriptor. IEEE TMI (Accepted) 5

16. Karaçhali, B., Davatzikos, C.: Simulation of tissue atrophy using a topology preserving transformation model. IEEE TMI 25(5), 649-652 9 (2006) 